\title{
Downstream of GA4, PbCYP78A6 Regulates Parthenogenesis by Mediating Cell Cycle-Related Genes in Pear (Pyrus Bretshneider Rehd.)
}

\section{Haiqi Zhang}

Northwest A\&F University: Northwest Agriculture and Forestry University

\section{Wei Han}

Northwest Agriculture University: Northwest Agriculture and Forestry University

\section{Huibin Wang}

Northwest Agriculture University: Northwest Agriculture and Forestry University

Liu Cong

Northwest Agriculture University: Northwest Agriculture and Forestry University

\section{Rui Zhai}

Northwest Agriculture University: Northwest Agriculture and Forestry University

\section{Chengquan Yang}

Northwest Agriculture University: Northwest Agriculture and Forestry University

\section{Zhigang Wang}

Northwest Agriculture University: Northwest Agriculture and Forestry University

Lingfei Xu ( $\square$ lingfxu2013@sina.com )

Northwest Agriculture University: Northwest Agriculture and Forestry University

\section{Research article}

Keywords: parthenocarpy, pollination, GA4, PbCYP78A6, fruit development.

Posted Date: April 13th, 2021

DOI: https://doi.org/10.21203/rs.3.rs-409647/v1

License: (c) (1) This work is licensed under a Creative Commons Attribution 4.0 International License. Read Full License 


\section{Abstract}

Background: Parthenocarpy results in traits attractive to both consumers and breeders, and it overcomes the obstacle of self-incompatibility in the fruit set of horticultural crops, including pear (Pyrus bretshneider). However, there is limited knowledge regarding the genetic and molecular mechanisms that regulate parthenogenesis.

Results: Here, in a transcriptional comparison between pollination-dependent and $\mathrm{GA}_{4}$-induced parthenocarpy, PbCYP78A6 was identified and proposed as a candidate gene involved in parthenocarpy. PbCYP78A6 is similar to Arabidopsis thaliana CYP78A6 and is highly expressed in pear hypanthia. The increased $P b C Y P 78 A 6$ expression, as assessed by RT-qPCR, was induced by pollination and $\mathrm{GA}_{4}$ exposure. The ectopic overexpression of $P b C Y P 78 A 6$ contributed to parthenocarpic fruit production in tomato. The $P b C Y P 78 A 6$ expression coincided with fertilized and parthenocarpic fruitlet development and the expression of fruit development-related genes as assessed by cytological observations and RTqPCR, respectively. PbCYP78A6 RNA interference and overexpression revealed that the gene is an upstream regulator of fruit development-related genes in pear.

Conclusions: Our findings indicate that $P b C Y P 78 A 6$ plays a critical role in cell proliferation and provide insights into controlling parthenocarpy.

\section{Background}

Angiosperms have evolved double-fertilization processes, which require coordinated communication between gametophytic and sporophytic tissues, and fruit development as pivotal steps of their survival and dispersal strategies [1-2]. Fruit initiation requires successful pollination and fertilization, but parthenocarpy uncouples this synchronized association and triggers fruit development [3]. Phytohormones are thought to be triggers induced by fertilization. Many strategies to produced virgin fruit include the exogenous application or overproduction of plant hormones, particularly auxins and gibberellins (GAs) [4-6], as well as the mutation of specific genes in these two plant hormone signaling pathways [7-8]. Auxins partially act upstream of GAs in inducing fruit set [9]. Complex mechanisms involving different hormones have been revealed, but there is limited knowledge regarding the mechanisms that underlie parthenocarpy.

Parthenocarpy results in traits attractive to both consumers and breeders. It also overcomes the obstacle of self-incompatibility in the fruit set of horticultural crops, including pear (Pyrus bretshneider) [3]. The fleshy fruit of pear is derived from the hypanthium and is known as an accessory fruit. Owing to the traits of perennial fruit trees, knowledge regarding the mechanism behind parthenocarpy in pear is still limited. In recently years, research has mainly focused on exogenous applications of plant hormones to induce parthenocarpy in pear [10-14], whereas the genetic mechanisms underlying parthenocarpy are rarely studied. 
Overexpressing CYP78A9, a member of cytochrome P450 78A subfamily, identified by a transfer DNA activation-tagging screen, produces the parthenocarpic phenotype in Arabidopsis thaliana [15]. CYP78A6 acts redundantly with CYP78A9 to control reproductive organ development [16]. To date, the catalytic function of CYP78A enzymes remain largely unknown, but the expression patterns and effects of related genes have been widely elucidated [17-19]. Overexpressing members of the CYP78A family increases non-autonomous cell proliferation and the sequential formatting of large organs [20-21]. The growth and development of multicellular organisms is controlled by cell-cycle progression, which is mediated by the periodic activation of complexes containing cyclins (CYCs) and cyclin-dependent kinases (CDKs) [22] The functions of CYP78A family members and their roles in parthenocarpy have not been reported, nor have correlations between their expression levels and cell proliferation-related genes in pear.

In this work, we provide a detailed description of $P b C Y P 78 A 6$ 's functions in pear parthenogenesis. We produced matured parthenocarpic fruit from pollination-dependent and $\mathrm{GA}_{4}$-induced 'Dangshansu' pear. Using comparative transcriptome and qRT-PCR analyses, we determined that PbCYP78A6 expression was intimately correlated with fruit set and development. Stable transgenes in tomato demonstrated the contribution of $P b C Y P 78 A 6$ to parthenogenesis. The overexpression and RNA interference of $P b C Y P 78 A 6$ in pear calli revealed that the gene is an upstream activator of cell proliferation. Thus, $P b C Y P 78 A 6$, induced by $\mathrm{GA}_{4}$, regulates parthenogenesis by mediating cell proliferation-related genes.

\section{Results}

\section{A transcription analysis identified a cytochrome P450 gene, PbCYP78A6, potentially responsible for parthenocarpy}

The $\mathrm{GA}_{4}$ treatments resulted in a relatively high fruit set rate of $93.2 \%$ and induced seedless fruits with decreased weights and increased fruit indices and hardness levels compared with seeded fruits, whereas $\mathrm{GA}_{3}$ treatments induced a relatively low fruit set rate of $56.8 \%$ and did not induce mature fruit formation (Table 1; Additional file 1: Fig. S1).

Table 1

The effects of $\mathrm{GA}_{4}$ and $\mathrm{GA}_{3}$ on 'Dangshansu' pear fruit set

\begin{tabular}{|c|c|c|c|c|}
\hline Treatment & UP & HP & $\mathrm{GA}_{4}$ & $\mathrm{GA}_{3}$ \\
\hline Fruit set rate & od & $87.9 \pm 2.2 b$ & $93.2 \pm 2.4 a$ & $47.0 \pm 1.6 \mathrm{c}$ \\
\hline \multicolumn{5}{|c|}{$\begin{array}{l}\text { UP un-pollinated, } \mathrm{HP} \text { hand pollinated, } \mathrm{GA}_{4} \mathrm{GA}_{4} \text { treatments, } \mathrm{GA}_{3}, \mathrm{GA}_{3} \text { treatments. Significant } \\
\text { differences among treatments as determined by one-way } \mathrm{ANOVA}(\mathrm{P}<0.05) \text { are indicated using } \\
\text { different lowercase letters }(a, b, c, d) \text {. The results represented are means of three biological replicates } \\
( \pm \mathrm{SD}, \mathrm{n}=3) \text {. }\end{array}$} \\
\hline
\end{tabular}

To further analyze the molecular mechanisms underlying parthenocarpy, an RNA-seq analysis was used to identify potential related genes. PbCYP78A6 (LOC103964254) was commonly up-regulated in pollinated and $\mathrm{GA}_{4}$-treatment groups (Additional file 2: Fig. S2; Additional file 3: Supplementary Table S1). 
A phylogenetic analysis demonstrated that PbCYP78A6 in $P$. bretshneider displayed very high similarity levels to AtCYP78A9 and AtCYP78A6 (Fig. 1). PbCYP78A6 was highly expressed in the sepal, which is an important component of pear fruit (Fig. 2A). During the early process of fruit set, PbCYP78A6's expression level significantly increased in the effective treatment groups, including hand-pollinated and $\mathrm{GA}_{4}$-treated (Fig. 2B). We separated the developing fruit into hypanthium, ovary wall and ovule (Fig. 2C). A relatively high $P b C Y P 78 A 6$ expression level was detected in the hypanthium compared with the ovary wall and ovule (Fig. 2D). We speculated that PbCYP78A6 expression correlated with fruit development and parthenogenesis in pear.

\section{PbCYP78A6 overexpression contributed to parthenocarpic fruit development in tomato}

To determine the potential roles of $P b C Y P 78 A 6$ in fruit development and parthenogenesis, transgenic tomato lines overexpressing the PbCYP78A6 gene were obtained (Additional file 4: Fig. S3). Under natural pollination conditions, transgenic tomato overexpressing $P b C Y P 78 A 6$ had significantly reduced numbers of seeds per fruit, and most of the fruits produced were seedless (Fig. 3A). At the mature stage, there were a few large seeds along with tracks of underdeveloped seeds in the transgenic fruits (Fig. 3B).

Observations and a statistical analysis of seeds determined that overexpressing PbCYP78A6 reduced the number of seeds per fruit (Fig. $3 C$ ). Seeded fruits were produced by pollinated ovaries in wild-type (WT) lines (Fig. 3D). The ovaries of PbCYP78A6-overexpression (OE) lines continued to develop and produce parthenocarpic fruits after being emasculated (Fig. 3D, E), whereas WT lines did not produce fruits without pollination. Thus, overexpressed $P b C Y P 78 A 6$ was involved in the regulation of fruit development and parthenocarpy.

\section{Cell division and expansion were induced as PbCYP78A6 expression increased}

CYP78A6 overexpression results in the production of large fruit owing to increased cell proliferation [16, 18]. To explore the cellular changes after $P b C Y P 78 A 6$ expression was highly induced, fruitlets were embedded in paraffin and sectioned. Phenotypic observations of the fruitlet sections were recorded (Fig. 4). The ovaries of the pollinated and $\mathrm{GA}_{4}$-treated groups were larger than those of the un-pollinated and $\mathrm{GA}_{3}$-treated groups (Fig. 4A-D). The thicknesses of the calyxes in $\mathrm{GA}_{4}$-treated and pollinated samples were significantly greater than those of the un-pollinated and $\mathrm{GA}_{3}$-treated groups (Fig. $4 \mathrm{E}-\mathrm{K}$ ). Compared with the un-pollinated treatment, hand pollination and $\mathrm{GA}_{4}$ exposure induced increased celllayer production and cell-area enlargement (Fig. 4J, K). Thus, the increased PbCYP78A6 expression activated cell division and expansion.

\section{The expression of fruit development-related genes was activated during parthenogenesis}

To further understand the molecular mechanisms underlying parthenogenesis, the dynamic growth and correlated transcript levels of cell expansion- and division-related genes were observed in 'Dangshansu' fruitlets at an early stage. Notably, $\mathrm{GA}_{4}$-treated and pollinated fruitlets underwent rapidly growth, whereas 
$\mathrm{GA}_{3}$-treated and un-pollinated fruitlets did not show significant growth-related changes (Fig. 5A). Moreover, un-pollinated fruitlets showed growth retardation. On the basis of our previous studies [10-12], expansin-A4 (EXPA4), cyclinA2-4, G2/mitotic-specific cyclin-2-like (CCNB2L), cyclin-dependent kinase B2-2 (CDKB22), Pbcyclin-dependent kinase B2-2-like (CDKB22L) and cyclin-dependent kinase inhibitor 6-like (CDKI6L) were screened, and their expression levels played critical roles in fruit development. The RTqPCR results showed that as the morphological changes occurred, the expression levels of these genes were significantly greater in pollinated and $\mathrm{GA}_{4}$-treated fruitlets than in un-pollinated and $\mathrm{GA}_{3}$-treated fruitlets (Fig. 5B). The CDKI6L expression level was repressed more by $\mathrm{GA}_{4}$ than $\mathrm{GA}_{3}$ (Fig. 5B). Intriguingly, the $P b C Y P 78 A 6$ expression pattern was almost consistent with the those of fruitlet development-related genes (Figs. 2 and 5). We speculated that PbCYP78A6 acts upstream of cell proliferation-related genes to regulate fertilized and parthenocarpic fruit growth.

\section{PbCYP78A6 was involved in the regulation of fruit development-related genes}

To further characterize the correlations between $P b C Y P 78 A 6$ and parthenogenesis-related genes, transgenic PbCYP78A6-OE and -RNA interference (RNAi) calli were generated. Green fluorescent protein (GFP) signals were detected in PbCYP78A6-OE and -RNAi lines (Fig. 6A). RT-qPCR was used to identify changes in PbCYP78A6 expression (Fig. 6B, C). In PbCYP78A6-RNAi calli, PbExpA4 and PbCDKI6L expression levels were extremely significantly increased (Fig. 6B), and the $P b C D K B 22, P b C D K B 22 L$, $P b C C N B 2 L$ and $P b C y c l i n A 24$ transcript levels were also increased (Fig. 6B; Additional file 5: Fig. S4), compared with in WT. However, in PbCYP78A6-OE calli, the positive regulators of cell proliferation, including $P b E x p A 4, P b C D K B 22, P b C D K B 22 L$ and $P b C y c l i n A 24$, were significantly up-regulated, while a negative regulator of cell proliferation, $P b C D K I 6 L$, was down-regulated compared with in WT (Fig. 6C; Additional file 5: Fig. S4). Thus, $P b C Y P 78 A 6$ regulated cell proliferation-related genes involved in fruit growth.

\section{Discussion}

In higher plants, an ovary can either be fertilized and subsequently grow into a fruit, or, owing to the lack of successful fertilization, it can enter the abscission process. Exogenous applications of multiple hormones mimic fertilization functions and fruit set, independently of fertilization, resulting in parthenocarpy $[9,23]$. In 'Dangshansu' pear, $\mathrm{GA}_{4}$ is effective in inducing parthenocarpy [10-12, 24], whereas $\mathrm{GA}_{3}$ does not induce parthenocarpy. Here, the difference between $\mathrm{GA}_{4}$ - and $\mathrm{GA}_{3}$-induction in pear was assessed to investigate the mechanisms of parthenocarpy-related downstream hormones.

In a comparison of transcriptomes between pollination-dependent and parthenocarpic fruit set, PbCYP78A6 was identified as a candidate gene involed in the regulation of parthenogenesis. PbCYP78A6 is similar to AtCYP78A6 and AtCYP78A9 (Fig. 1), but its expression pattern differed from those of AtCYP78A6 and AtCYP78A9 [16, 21]. PbCYP78A6 was determined to be widely expressed in reproductive organs, but it was highly expressed in the sepal (Fig. 2A), which partly develops into an accessory fruit in pear. PbCYP78A6 expression was significantly activated by $\mathrm{GA}_{4}$ and pollination, and the expression level 
increased as the fruit set and developmental processes continued (Fig. 2B), which is consistent with AtCYP78A9 activation being detected in developing seeds, septa, funiculi and placental tissues following fertilization [21]. Overexpressing AtCYP78A6 or AtCYP78A9 promotes the growth of not only seeds, but also the reproductive tissues, including sepals and siliques [16, 21], which indicates that AtCYP78A6 and AtCYP78A9 control their development. In pear, the cells of the exocarp are capable of cell division, in which new cell layers arise owing to periclinal cell division. In our study, PbCYP78A6 expression was rarely detected in ovules, but occurred at a high level in the pericarp and hypanthium, in which cell division occurs vigorously (Fig. 2C, D). This indicated that PbCYP78A6 is involved in pear fruit development. Thus, the different structures between fruit and silique hint at an important role for $P b C Y P 78 A 6$ in parthenogenesis.

Under normal pollination conditions, the flowers of PbCYP78A6-OE transgenic plants produced fruits with reduced seed numbers and even some seedless fruits (Fig. 3). This reproductive organ phenotype was similar to that seen in Arabidopsis overexpressing AtCYP78A6 [16]. The auxin production and GAmediated responses of ovules are dependent on pollination events that trigger fruit development [25]. Fruit development occurred normally in transgenic lines having low numbers of, and even no, seeds (Fig. 3A-C), which demonstrated that fruit generation occurred independently of the fertilization signals in PbCYP78A6-OE plants. Further emasculation experiments demonstrated that the flowers of transgenic plants had the capability of producing parthenocarpic fruits (Fig. 3D, E). In Arabidopsis, large and seedless fruit have also been induced by the overexpression of CYP78A9 [18]. Here, we report that like AtCYP78A9, PbCYP78A6 was capable of inducing parthenocarpic fruit set and development in tomato.

Silencing the Arabidopsis CYP78A6/EOD3 homolog PaCYP78A6 decreases fruit size by affecting cell proliferation [19], indicating that $P a C Y P 78 A 6$ acts upstream of cell division and expansion. Thus, the parthenocarpy produced by overexpressing PbCYP78A6 may also arise from the activation of cell division and expansion. The differential expression of $P b C Y P 78 A 6$ was consistent with the significantly increased cell division and expansion that occurred in the tissue-containing calyx and mature ovary compared with those of the un-pollinated group (Fig. 2). The process of fruit set is accompanied by cell division and expansion [26], indicating that $P b C Y P 78 A 6$ plays an essential role in fruit formation.

Fruit development is largely dependent on cell division and expansion, and cell division is governed by two gene families, CDKs and CYCs [27]. Two other families, EXPA and EXPB, have the ability to regulate cell expansion by extending cell walls [28]. CDKBs, CYCAs and EXPAs participate in regulating fruit development [29-31]. In accordance with our previous study [10,12], the expression levels of selected cell division- and expansion-related genes were analyzed during the early fruit set stage (Fig. 5). Among them, PbExpA4, PbCyclinA2-4, PbCCNB2L, PbCDKB22 and PbCDKB22-like had up-regulated expression pattrens, which agreed with the histological observations (Fig. 4). Moreover, a CDK inhibitor gene, CDKI6L, was identified, and its expression decreased in $\mathrm{GA}_{4}$-treated fruitlets. However, the $\mathrm{GA}_{3}$ treatment failed to repress the expression of CDKI6L. ICK1 and ICK2 expression reduce the CDK activity and affect cell division in $A$. thaliana [32], indicating that the inhibition of CDKI6L also plays an important role in fruit set. Thus, these genes are important for pear fruit development. Intriguingly, PbCYP78A6 expression was 
almost consistent with the expression patterns of PbEXPA4, PbCyclinA2-4, PbCCNB2L, PbCDKB22 and $P b C D K B 22-l i k e$, and it was negatively correlated to the expression of the repressor CDKI6L (Figs. 2B and $4 \mathrm{~B})$.

PaCYP78A9's effect on plant organ size is regulated by cell cycle-related genes [19], whereas $P b C Y P 78 A 6$ 's effect on fruit development was mediated by specific fruit development-related genes in pear. In PbCYP78A6-RNAi pear calli, silencing PbCYP78A6 did not halt cell proliferation (Fig. 5B; Additional file 5: Fig S4A). Overexpressing PbCYP78A6 promoted the expression of fruit growth-related genes and particularly repressed $P b C D K I 6 L$ expression in pear calli (Fig. 5C; Additional file 5: Fig S4B). The PbCDKB22, PbCDKB22-like, PbCyclinA2-4 and PbCCNB2L expression levels were negatively correlated with the PbCYP78A6 expression level. Similarly, silencing PaCYP78A6 or PaCYP78A9 does not completely repress the expression of all the cell proliferation-related genes [19, 33]. The evidence indicates that there must be other factors involved in controlling cell proliferation. We also identified a CDK repressor, $P b C D K I 6 L$, which was increasingly induced as PbCYP78A6 expression decreased (Fig. 5). The reduced CDK activity was attributed to the increased ICK1 expression, which represses cell division [32]. Thus, $P b C D K I 6 L$ plays a key role in the regulation of $P b C Y P 78 A 6$ expression in fruit development. Cell-wall loosening is critical for rapid cell division, and it is most often controlled by EXPs [34]. Both silencing and overexpressing PbCYP78A6 significantly promoted PbExpA4 expression, perhaps owing to the consequences of cell proliferation. The effects of PbCYP78A6 on cell cycle-related genes and parthenogenesis were confirmed, but the catalytic function of the PbCYP78A6 enzyme remains largely unknown. Thus, our findings indicate that $P b C Y P 78 A 6$ acts as an upstream regulator of fruit development during parthenogenesis (Fig. 7).

\section{Conclusions}

PbCYP78A6 was induced by pollination and $\mathrm{GA}_{4}$ treatments, and its overexpression resulted in parthenocarpic tomato. The effects of $P b C Y P 78 A 6$ on fruit development were mediated by cell cycledrelated genes. The utilization of CYP78A6 by fruit trees and its altered expression in fruits might provide a method for producing seedless fruits and enhancing crop yields (Fig. 7). The further elucidation of unknown plant growth-related substances may contribute to their utilization in regulating fruit development.

\section{Methods}

\section{Plant material and growth conditions}

Treated 'Dangshansu' pear trees (Pyrus bretshneider Rehd.) were planted in Pear experimental base of Northwest A\&F University located in MeiXian County, Shaanxi Province, China $\left(34.28^{\circ} \mathrm{N}, 108.22^{\circ} \mathrm{E} ; 562\right.$ $\mathrm{m}$ ). The average annual precipitation is $574.6 \mathrm{~mm}$, and the average annual temperature was $12.7^{\circ} \mathrm{C} .16-$ year-old 'Dangshansu' pear trees grafted onto Pyrus betulifolia Bge rootstocks were used as the experimental materials. Permissions for all the materials used in this experience have been obtained. 
Micro-Tom (Solanum lycopersicum L.) was chose for transformation in this experiment. Seeds were received from the gift of Xiangqiang Zhan, from Northwest A\&F University, Yangling, China. For transgenic experiments, seeds were soaked with sterile water and sterilized with $2 \%$ Sodium hypochlorite solution. Sterilized seeds were germinated and grown in glass jars covered with transparent lips, containing $30 \mathrm{ml}$ medium [Murashige and Skoog (1/2 MS) salts, $1.5 \%(\mathrm{w} / \mathrm{v})$ sucrose, and $0.74 \%(\mathrm{w} / \mathrm{v})$ agar], then put in a culturing chamber at $25^{\circ} \mathrm{C}$ under 16 -h/8-h light/dark conditions for 6-8 days until cotyledons were fully extended, which were used for next transformation experiments.

\section{Experimental treatments and samples}

Two days before anthesis, all of the treatments and the control were bagged to avoid pollination. To avoid the disturbing fruit set caused by natural pollination, the opened flowers and the weak buds were removed to make sure every single pear flower keeping consistent and unopened when the exogenous hormones were applied. $\mathrm{GA}_{3}$ solutions $50 \mathrm{mg} \mathrm{L}^{-1}$ and $\mathrm{GA}_{4}$ solutions of $50 \mathrm{mg} \mathrm{L}^{-1}$ were independently sprayed on individual un-pollinated flowers of 'Dangshansu' pears at anthesis. 1:1 water: ethanol mixture was sprayed on un-pollinated flowers as the un-pollinated treatment. Three branches of each treatment were used as three replicates. Fruitlets with complete structure per treatment were randomly sampled. Fruits at $0,3,4,6,9,30$ and 145 DAA were harvested for morphological observation and other experiments, respectively. After removing the stalks, sepal, stamens an gynoecium, fruitlets left sampled at 4 and 6 days after anthesis (DAA) were used for RNA sequencing and immediately fixed in formaldehyde-acetic acid-alcohol for paraffin section, respectively. Fruitlets at 0, 3, 6, 9 DAA were used for the analysis of gene expression patterns with the same dispose as above. Fruitlets with hand pollination at 3 DAA were subdivided into fruit stem, sepal, petal, stamen, stigma, ovary, and ovule for tissue-specific expression. Fruits at 30 DAA were subdivided into pericarp, hypanthium, ovary wall and ovule for the expression position of PbCYP78A6 in fruits. Each sample was harvested from 12 fruitlets, pooled, directly frozen in liquid nitrogen and stored at $-80^{\circ} \mathrm{C}$.

\section{Determination of fruit set rate}

A total of 30 blooms on each three branches was labeled and bagged immediately after receiving treatments. At 20 DAA, the bags were removed. The formula used to calculate the fruit-set rate was as follows:

Fruit set $(\%)=($ number of fruitlets remaining/number of total flowers counting $) \times 100$.

\section{Paraffin sectioning and data statistics}

To conduct the histological observations, fruit samples of four treatments in 'Dangshansu' pear were collected at 6 DAA when the significant changes were observed, immediately fixed in formaldehydeacetic acid-alcohol fixative [35] and stored at $4{ }^{\circ} \mathrm{C}$. The ovaries were dehydrated in an ethanol/xylene series and embedded in paraffin. They were then cut into 8- $\mu \mathrm{m}$-thick slices, dried and stained with safranin and fast green. The anatomical images were observed using a microscopic imaging system (BX51 + PD72 + IX71, OLYMPUS, Japan). Cell area and calyx thickness were calculated using Image J 
software (https://imagej.net/Welcome). Cell area calculation method was as follows: first, a certain area was randomly circled and the total cell area was calculated; second, the total number of cells was counted; and third, the representative cell area of a single cell was calculated as the total cell area divided into the total number of cells. All calculations were performed on more than three replicates.

\section{Transcription analysis}

To further understand the potential molecular mechanism underlying parthenocarpy, Samples from unpollinated, hand pollinated, $\mathrm{GA}_{3}$-treated (without pollination) and $\mathrm{GA}_{4}$-treated (without pollination) at 4 DAA were utilized for RNA sequencing. Significant morphological changes happened from 3 DAA to 6 DAA, so fruitlets at 4 DAA were selected to conduct RNA-seq experiment. Three independent biological replications were sequenced and analyzed. 12 samples (Un-pollination, Hand pollination, $\mathrm{GA}_{3}$ and $\mathrm{GA}_{4}$ treated samples of 'Dangshansu' pear at 4 DAA with three biological replicates, respectively) were subjected to total RNA extraction and Illumina HiSeq TM sequencing. Generating at least 40.1362.70 million raw reads among each library was followed by filtering process. After then, 39.7761.99 million clean reads remained with a Q30 percentage (an error probability lower than $0.1 \%$ ) ranging from 90.77 to $95.17 \%$. The clean reads accepted were mapped to the reference pear ( $P$. bretschneideri Rehd.) genome by using HISAT software. Range from $72.35 \%-80.15 \%$ of total reads were mapped to the reference genome (Additional file 6: Supplementary Table S2). Over $95.9 \%$ of reads mapped to genome were mapped to genomic exon regions. FPKM (expected number of Fragments Per Kilobase of transcript sequence per Millions base pairs sequenced) was used to evaluate genes expression level. FPKM $>1$ was termed as standard to determine the expression of genes. 12 genes were selected to verify the reliability of transcription data by qRT-PCR (Additional file 7: Fig. S5). The RNA-Seq data of un-pollinated ovaries were used as the controls. padj $<0.05$ and $\mid \log 2$ (ratio) $\mid>1$ were used as the thresholds to determine the significance of DEGs. The DEGs were identified by pairwise comparisons of the 4 libraries, HP vs. UP, GA 4 vs. $\mathrm{GA}_{3}$ in 'Dangshansu' pear. Pearson correlation between biological replicates ranged from 0.9740.987. Gene was annotated using the 'Dangshansuli' (http://www.ncbi.nlm.nih.gov/genome/? term=pyrus) database as a reference. Venn diagram analysis was used to perform differential expression analysis. Kyoto Encyclopedia of Genes and Genomes (KEGG) functional annotations were based on sequence homologies against public database (www.genome.jp/kegg/). The expression profiles of DEGs were performed on TB-tools [36].

\section{Real-time Quantitative PCR (RT-qPCR) validation of gene expression levels}

The qRT-PCR was performed on a Step One plus Real-Time PCR Instrument (Thermo Fisher Scientific, Massachusetts, USA ) machine using TB Green premix Ex Taq II Kit (Takara, Dalian, China). About $0.3 \mathrm{~g}$ samples were weighed and quickly grinded to powder in liquid nitrogen. Total RNA was extracted using a Polysaccharides and Polyphenolics-rich RNAprep Pure Plant kit (Tiangen, Beijing, China). RNA concentration and quality were assessed by spectrophotometry on Thermo Scientific Microplate Reader (multiscan GO) and Polypropylene gel electrophoresis on electrophoresis meter (DYY-6D), respectively. 
cDNA was synthesized by the reversely transcription of $1 \mu \mathrm{g}$ total RNA using a PrimeScript RT reagent kit with gDNA Eraser (Takara, Dalian, China). Primers of target genes were designed by Primer Premier 5.0 software (PREMIER Biosoft) and NCBI Primer-BLAST tools (https://www.ncbi.nlm.nih.gov/tools/primerblast/index.cgi). The Actin 7 gene was used as the internal reference for the gene expression analysis. Primers for verification of transcription data were described in Additional file 8: Supplementary Table S3. Primers for PbCYP78A6 (LOC103964254), Pbcyclin-dependent kinase B2-2 (LOC103961775), Pbcyclindependent kinase B2-2like (LOC103952922), Pbexpansin-A4 (LOC103951053), G2/mitotic-specific cyclin2-like (CCNB2L) (LOC103962422), PbCyclinA2-4 (LOC103931294), PbCDKI6-like (LOC103964480) were listed in Additional file 8: Supplementary Table S3. The PCR reactions were carried out using an initial incubation at $95^{\circ} \mathrm{C}$ for $30 \mathrm{~s}$, and then 40 cycles of $95^{\circ} \mathrm{C}$ for $5 \mathrm{~s}$ and $60^{\circ} \mathrm{C}$ for $30 \mathrm{~s}$. All reactions were performed on three biological and three technical replicates. Relative quantification of specific mRNA levels was performed using the cycle threshold (Ct) $2^{-\triangle \Delta C T}$ method [37].

\section{Phylogenetic analysis}

The full-length CDS of PbCYP78A6 (LOC103964254) was isolated from 'Dangshansu' pear (http://www.ncbi.nlm.nih.gov/genome/?term = pyrus). Amino acid sequences of PbCYP78A6 and other CYP78A subgroup members of other plants were aligned using ClustalW [38]. MEGA5.10 was applied to construct the phylogenetic tree with the neighbor-joining statistical method. In addition, 1000 bootstrap replications were performed for testing of phylogeny [39].

\section{Construction of plasmids and generation of transgenic pear calli}

To generate transgenic pear calli, the coding region of $P b C Y P 78 A 6$ was cloned using primers PbCYP78A6-OE F/R and introduced into the vectors pGWB414 vector based on gateway recombination technology (Invitrogen) to create an overexpression vector. The vectors pHellsgate2 and pK7WIWG2D were used as RNAi-mediated vectors for silencing PbCYP78A6 as described [40, 41]. The vectors were transformed into Agrobacterium tumefaciens strain EHA105 for infiltration.

The induction of pear calli and their transformation were described previously [42]. Briefly, the prefabricated suspension of EHA105 was incubated with 15-day-old pear calli for $15 \mathrm{~min}$. After cocultured on MS medium containing $1.0 \mathrm{mg} \cdot \mathrm{L}^{-1}$ of $2,4-\mathrm{D}$ and $0.25 \mathrm{mg} \cdot \mathrm{L}^{-1}$ of $6-\mathrm{BA}$ for 2 days at $24^{\circ} \mathrm{C}$. Subsequently, the calli were washed three times with sterile water containing $300 \mathrm{mg} \cdot \mathrm{L}^{-1}$ of Cefotaxime sodium and transferred to MS medium supplemented with $300 \mathrm{mg} \cdot \mathrm{L}^{-1}$ of Cefotaxime and $50 \mathrm{mg} \cdot \mathrm{L}^{-1}$ of kanamycin sulfate for transgene selection.

\section{Production of transgenic lines in tomato}

The full-lenth PbCYP78A6 (LOC103964254) coding sequence (CDs) was isolated from 'Dangshansu' cDNA using primers with adaptors (Additional file 9: Supplementary Table S4) designed by Snap Gene software 1.1.3, and then cloned into the $\mathrm{BamH} /$ and Sac/ restriction enzyme sites downstream of a Cauliflower mosaic virus (CaMV) 35S promoter in the pBI121 vector to generate a overexpression 
construct using a ClonExpress One Step Cloning kit (Vazyme, Nanjing, China). The recombinant vect was transformed into Agrobacterium tumefaciens strain LBA4404 by heat-shock method, and the positive monoclonal Agrobacterium cell proliferated at $28^{\circ} \mathrm{C}, 200 \mathrm{rpm}$, dark in Luria-Bertani (LB) solid medium containing appropriate antibiotics (kanamycin and rifampicin). After incubation for appropriate time, the Agrobacterium cell concentration achieving to $\mathrm{OD}_{600} \approx 0.5-0.8$ were centrifuged at $600 \mathrm{rpm}$, collected in the tube, and then suspended in MS isopyknic to LB solution. The bacterial suspension was used for tomato transformation.

Transformation assays were carried out as previously described [43]. Briefly, sterilized tomato seeds were grown until its cotyledons full stretched in glass jars. Cotyledons were cut into sections and placed on Petri dishes containing solidified MS1 medium for 2 days in the dark, Then immersed them in bacterial suspensions prepared above for $10 \mathrm{~min}$. Explants impregnated with bacterial suspension were blotted with filter paper, then cultured in the dark for 2 days in MS1 medium. Then the explants were transferred to MS2 solidified medium. After explants developed resistant calli produced shoots, 1-2 $\mathrm{cm}$ shoots were excised and placed on MS3 medium in glass jars. After took root, explants with root were cultured in pots containing vermiculite, watered with Hoagland's solution, and conditioned in a growth chamber before transferring to the greenhouse. Progeny from the transgenic plants were obtained by selfing under controlled conditions.

Medium used above were as follow: Suspension liquid included MS salts supplemented with $0.4 \mathrm{mg} \mathrm{I}^{-1}$ thiamine hydrochloride, $100 \mathrm{mg} \mathrm{I}^{-1}$ myo-inositol, $2 \%(\mathrm{w} / \mathrm{v})$ sucrose and $200 \mu \mathrm{M}$ acetosyringone. MS1 contained MS salts supplemented with vitamins, $3 \%(\mathrm{w} / \mathrm{v})$ sucrose, $100 \mathrm{mg} \mathrm{I}^{-1}$ myo-inositol, $4 \mathrm{mg} \mathrm{I}^{-1}$ indole acetic acid (IAA), $4 \mathrm{mg} \mathrm{I}^{-1}$ kinetin, and $0.8 \%(\mathrm{w} / \mathrm{v})$ agar. MS2 contained MS1 supplemented with 1 $\mathrm{mg} \mathrm{l}^{-1}$ zeatin, $300 \mathrm{mg} \mathrm{I}^{-1}$ cefatoxime, and $100 \mathrm{mg} \mathrm{I}^{-1}$ kanamycin. MS3 was consist of MS salts, $2 \%$ (w/v) sucrose, $100 \mathrm{mg} \mathrm{l}^{-1}$ myo-inositol, $1 \mathrm{mg} \mathrm{l}^{-1}$ thiamine, $0.1 \mathrm{mg} \mathrm{I}^{-1} \mathrm{IAA}$, and $0.8 \%(\mathrm{w} / \mathrm{v})$ agar.

\section{Abbreviations}

$\mathrm{GA}_{4}$ : gibberellin A4; $\mathrm{GA}_{3}$ : gibberellin A3; HP: Hand Pollination; UP: Un-pollination; OE: Over-Expression; EXPA4: Expansin-A4; CCNB2L: G2/mitotic-specific cyclin-2-like; CDKB22: Cyclin-dependent kinase B2-2; CDKB22L: PbCyclin-dependent kinase B2-2-like; CDKI6L: cyclin-dependent kinase inhibitor 6-like; RNAi: RNA interference; SD: Standard Deviation.

\section{Declarations}

Ethics approval and consent to participate: Not applicable.

Consent for publication: Not applicable

Availability of data and materials: Permissions for all the materials used in this experience had been obtained. No further permission was therefore needed. All data generated and analyzed during this study 
are included in this published article. Extra data has been appended as supplementary Tables. All the genes' sequence and information can be accessed on National Center of Biotechnology Information (https://www.ncbi.nlm.nih.gov/).

Competing interests: The authors declare no conflict of interest.

Funding: This research was funded by the National Key R\&D Program of China (2019YFD1001400) and Weinan Experimental Station foundation of Northwest A\&F University. Apart from providing financial support, funding agencies played no role in the design of the studies, the collection, analysis and interpretation of the data, and the writing of the manuscripts.

Authors' Contributions: HQZ carried out the experiments and wrote the initial draft of the manuscript. LFX, ZGW, RZ, CQY designed the experiments. HQZ, HBW, WH performed the experiments and analyzed the data. LFX, ZGW, RZ, CQY revised the manuscript. All authors have participated in this research and approved the final manuscript.

Acknowledgments: We thank Qingmei Guan, Prof., from Northwest A\&F University, Yangling, China, for providing pK7WIWG2D plasmid. We thank Xiangqiang Zhan, Prof., from Northwest A\&F University, Yangling, China, for generally providing tomato seeds and transgene method. We thank Lesley Benyon, PhD, from Liwen Bianji, Edanz Group China (www.liwenbianji.cn/ac), for editing the English text of a draft of this manuscript.

Authors' information: College of Horticulture, Northwest A\&F University, Taicheng Road NO.3, Yangling, Shaanxi Province, China. State Key Laboratory of Crop Stress Biology for Arid Areas, Northwest A\&F University, Taicheng Road NO.3, Yangling, Shaanxi Province, China

Conflicts of Interest: The authors declare no conflict of interest.

\section{References}

1. Berger F., Grini P. E. and Schnittger A. Endosperm: an integrator of seed growth and development. Curr Opin Plant Biol. 2006; 9(6): 664-670.

2. Dumas C. and Rogowsky P. Fertilization and early seed formation. C R Biol. 2008; 331(10): 715-725.

3. Joldersma D. and Liu Z. The making of virgin fruit: the molecular and genetic basis of parthenocarpy. J Exp Bot. 2018; 69: 955-962.

4. Garcia-Hurtado N., Carrera E., Ruiz-Rivero O., Lopez-Gresa M. P., Hedden P., Gong F. and Garcia-Martinez J. L. The characterization of transgenic tomato overexpressing gibberellin 20-oxidase reveals induction of parthenocarpic fruit growth, higher yield, and alteration of the gibberellin biosynthetic pathway. $J$ Exp Bot. 2012; 63(16): 5803-5813. 
5. Prosser M. V. and Jackson G. A. D. Induction of Parthenocarpy in Rosa arvensis Huds. with Gibberellic Acid. Nature. 1959; 184(4680): 108-108.

6. Rotino G. L., Perri E., Zottini M., Sommer H. and Spena A. Genetic engineering of parthenocarpic plants. Nat Biotechnol. 1997; 15(13): 1398-1401.

7. de Jong M., Wolters-Arts M., Feron R., Mariani C. and Vriezen W. H. The Solanum lycopersicum auxin response factor 7 (SIARF7) regulates auxin signaling during tomato fruit set and development. Plant $\mathrm{J}$. 2009; 57(1): 160-170.

8. Martí C., Orzáez D., Ellul P., Moreno V. and Granell A. Silencing of DELLA induces facultative parthenocarpy in tomato fruits. Plant J. 2008; 52(5): 865-876.

9. Serrani J. C., Ruiz-Rivero O., Fos M. and Garcia-Martinez J. L. Auxin-induced fruit-set in tomato is mediated in part by gibberellins. Plant J. 2008; 56(6): 922-934.

10. Cong L., Yue R., Wang H., Liu J., Zhai R., Yang J., Wu M., Si M., Zhang H., Yang C., Xu L. and Wang Z. 2,4-D-induced parthenocarpy in pear is mediated by enhancement of GA4 biosynthesis. Physiol Plantarum. 2019; 166(3): 812-820.

11. Liu J., Zhai R., Liu F., Zhao Y., Wang H., Liu L., Yang C., Wang Z., Ma F. and Xu L. Melatonin Induces Parthenocarpy by Regulating Genes in Gibberellin Pathways of 'Starkrimson' Pear (Pyrus communis L.). Front Plant Sci. 2018a; 9: 946.

12. Liu L., Wang Z., Liu J., Liu F., Zhai R., Zhu C., Wang H., Ma F. and Xu L. Histological, hormonal and transcriptomic reveal the changes upon gibberellin-induced parthenocarpy in pear fruit. Hortic Res. 2018b; 5: 1.

13. Niu Q., Wang T., Li J., Yang Q., Qian M. and Teng Y. Effects of exogenous application of GA4 + 7 and $\mathrm{N}$-(2-chloro-4-pyridyl)-N'-phenylurea on induced parthenocarpy and fruit quality in Pyrus pyrifolia 'Cuiguan'. Plant Growth Regul. 2015; 76(3): 251-258.

14. Zhang C., Lee U. and Tanabe K. Hormonal regulation of fruit set, parthenogenesis induction and fruit expansion in Japanese pear. Plant Growth Regul. 2008; 55(3): 231-240.

15. Meyerowitz T. I. a. E. M. Overexpression of a gene encoding a cytochrome P450, CYP78A9, induces large and seedless fruit in arabidopsis. Plant Cell. 2000; 12: 1541-1550.

16. Fang W., Wang Z., Cui R., Li J. and Li Y. Maternal control of seed size by EOD3/CYP78A6 in Arabidopsis thaliana. Plant J. 2012; 70(6): 929-939.

17. Anastasiou E., Kenz S., Gerstung M., MacLean D., Timmer J., Fleck C. and Lenhard M. Control of plant organ size by KLUH/CYP78A5-dependent intercellular signaling. Dev Cell. 2007; 13(6): 843-856. 
18. Ito T. and Meyerowitz E. M. Overexpression of a Gene Encoding a Cytochrome P450, CYP78A9, Induces Large and Seedless Fruit in Arabidopsis. Plant Cell. 2000; 12(9): 1541-1550.

19. Qi X., Congli L., Lulu S., Yuhong L. and Ming L. PaCYP78A9, a Cytochrome P450, Regulates Fruit Size in Sweet Cherry (Prunus avium L.). Front Plant Sci. 2017; 8: 2076.

20. Nikolai M., Adamskia., Elena A., Sven E., Carmel M., O'Neillc. and Michael L. Local maternal control of seed size by KLUH/CYP78A5-dependent growth signaling. P Natl Acad Sci U.S.A. 2009; 106(47): 2011520120.

21. Sotelo-Silveira M., Cucinotta M., Chauvin A. L., Montes R. A. C., Colombo L., Marsch-Martínez N. and Folter S. D. Cytochrome P450 CYP78A9 Is Involved in Arabidopsis Reproductive Development.Plant Physiol. 2013; 8(8): 779-799.

22. Inzé H. S. a. D. When plant cells decide to divide. Trends Plant Sci. 2001; 6(8): 359-364.

23. Muday G., Ding J., Chen B., Xia X., Mao W., Shi K., Zhou Y. and Yu J. Cytokinin-Induced Parthenocarpic Fruit Development in Tomato Is Partly Dependent on Enhanced Gibberellin and Auxin Biosynthesis. Plos one. 2013; 8(7): e70080.

24. Wang H., Wu T., Liu J., Cong L., Zhu Y., Zhai R., Yang C., Wang Z., Ma F. and Xu L. PbGA20ox2 Regulates Fruit Set and Induces Parthenocarpy by Enhancing GA4 Content. Front Plant Sci. 2020; 11: 113.

25. Dorcey E., Urbez C., Blazquez M. A., Carbonell J. and Perez-Amador M. A. Fertilization-dependent auxin response in ovules triggers fruit development through the modulation of gibberellin metabolism in Arabidopsis. Plant J. 2009; 58(2): 318-332.

26. de Jong M., Mariani C. and Vriezen W. H. The role of auxin and gibberellin in tomato fruit set. J Exp Bot. 2009; 60(5): 1523-1532.

27. Inze D. and De Veylder L. Cell cycle regulation in plant development. Annu Rev Genet. 2006; 40: (77105).

28. Cosgrove D. J. Growth of the plant cell wall. Nat Rev Mol Cell Bio. 2005; 6(11): 850-861.

29. Brummell. D. A., Harpster. M. H. and Dunsmuir P. Differential expression of expansin gene family members during growth and ripening of tomato fruit. Plant Mol Biol. 1999; 39: (161-169).

30. Czerednik A., Busscher M., Bielen B. A., Wolters-Arts M., de Maagd R. A. and Angenent G. C. Regulation of tomato fruit pericarp development by an interplay between CDKB and CDKA1 cell cycle genes. J Exp Bot. 2012; 63(7): 2605-2617. 
31. Joubes. J., Walsh. D., Raymond. P. and Chevalier. C. Molecular characterization of the expression of distinct classes of cyclins during the early development of tomato fruit. Planta. 2000; 211: (430-439).

32. Wang H., Zhou Y. M., Susan G., Whitwill S. and Fowke L. C. Expression of the plant cyclin-dependent kinase inhibitor ICK1 affects cell division, plant growth and morphology. Plant J. 2000; 24(5): 613-623.

33. Qi X., Liu C., Song L. and Li M. Arabidopsis EOD3 homologue PaCYP78A6 affects fruit size and is involved in sweet cherry (Prunus avium L.) fruit ripening. Sci Hortic. 2019; 246: 57-67.

34. Lee Y., Choi D. and Kende H. Expansins: ever-expanding numbers and functions. Curr. Opin. Plant Biol. 2001; 4: (527-532).

35. Henwood and Anthony. What is the Best Procedure to Remove Formalin Pigment from ForaldehydeAcetic Acid-Alcohol Fixed Tissues? J Histotechnol. 2010; 33(3): 109-111.

36. Chen C., Chen H., Zhang Y., Thomas H. R., Frank M. H., He Y. and Xia R. TBtools: An Integrative Toolkit Developed for Interactive Analyses of Big Biological Data Mol Plant. 2020; 13: (1-9).

37. Livak K. J. and Schmittgen T. D. Analysis of relative gene expression data using real-time quantitative PCR and the 2(-Delta Delta C(T)) Method. Methods. 2001; 25(4): 402-408.

38. Thompson. J. D., Gibson. T. J., Plewniak. F., Jeanmougin. F. and Higgins. D. G. The CLUSTAL_X windows interface: flexible strategies for multiple sequence alignment aided by quality analysis tools. Nucleic Acids Research. 1997; 25(24): 4876-4882.

39. Tamura K., Peterson D., Peterson N., Stecher G., Nei M. and Kumar S. MEGA5: molecular evolutionary genetics analysis using maximum likelihood, evolutionary distance, and maximum parsimony methods. Mol Biol Evol. 2011; 28(10): 2731-2739.

40. Xie Y., Chen P., Yan Y., Bao C., Li X., Wang L., Shen X., Li H., Liu X., Niu C., Zhu C., Fang N., Shao Y., Zhao T., Yu J., Zhu J., Xu L., van Nocker S., Ma F. and Guan Q. An atypical R2R3 MYB transcription factor increases cold hardiness by CBF-dependent and CBF-independent pathways in apple. New Phytol. 2018; 218(1): 201-218.

41. Zhou K., Hu L., Li Y., Chen X., Zhang Z., Liu B., Li P., Gong X. and Ma F. MdUGT88F1-Mediated Phloridzin Biosynthesis Regulates Apple Development and Valsa Canker Resistance. Plant Physiol. 2019; 180(4): 2290-2305.

42. Bai S., Tao R., Tang Y., Yin L., Ma Y., Ni J., Yan X., Yang Q., Wu Z., Zeng Y. and Teng Y. BBX16, a B-box protein, positively regulates light-induced anthocyanin accumulation by activating MYB10 in red pear. Plant Biotechnol J. 2019; 17(10): 1985-1997.

43. Ellul P., Garcia-Sogo B., Pineda B., Rios G., Roig L. A. and Moreno V. The ploidy level of transgenic plants in Agrobacterium-mediated transformation of tomato cotyledons ( Lycopersicon esculentum Mill.) 
is genotype and procedure dependent. Theor Appl Genet. 2003; 106(2): 231-238.

\section{Figures}

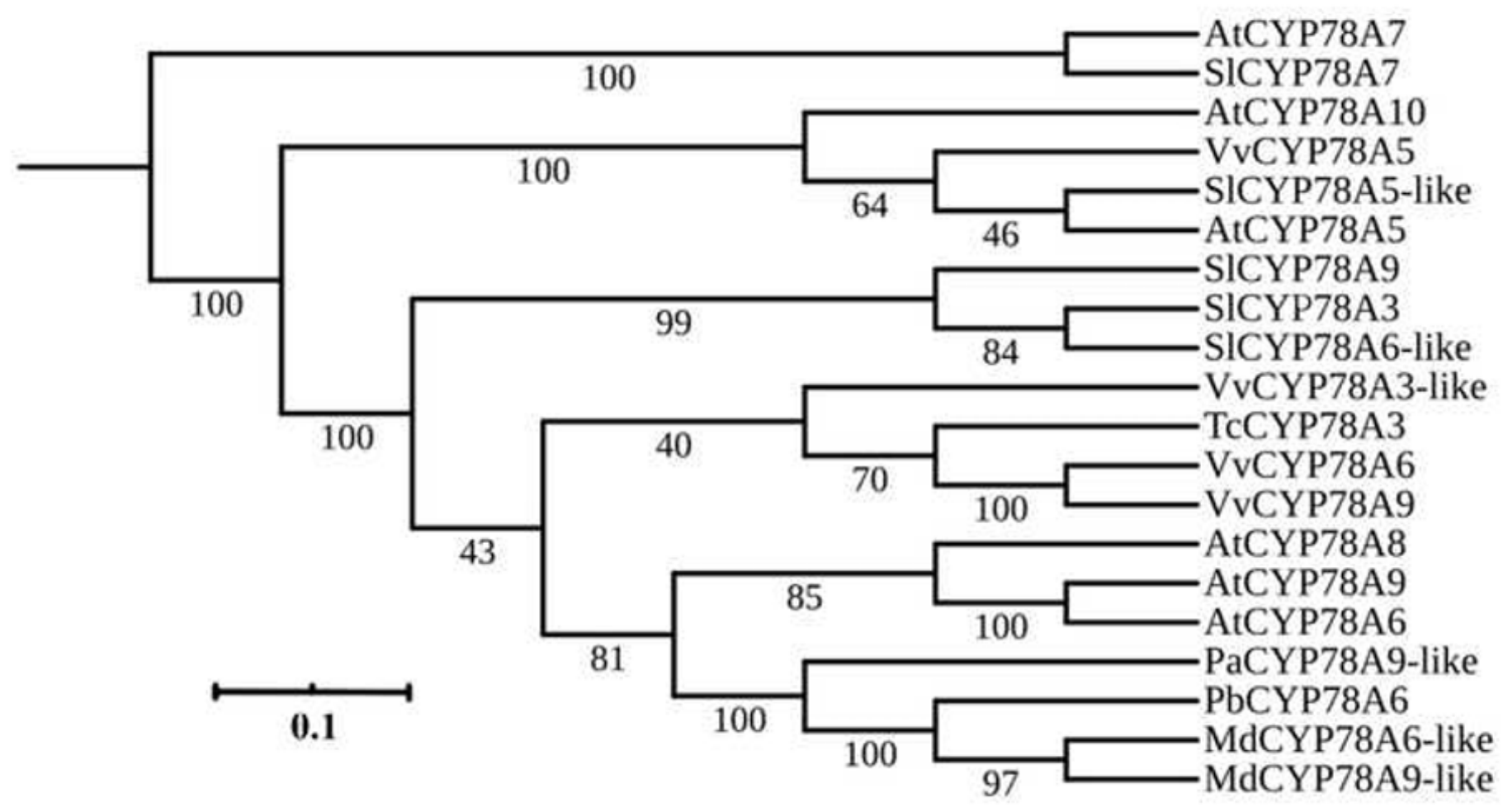

\section{Figure 1}

Phylogenic analysis of the PbCYP78A6 protein and CYP78A orthologs from other plant species. The protein accessions used were as follows: AtCYP78A7(Arabidopsis thaliana, NP_196559.1), SICYP78A7 (Solanum lycopersicum, XP_004252635.1), AtCYP78A10 (Arabidopsis thaliana, NP_177551.1), VvCYP78A5 (Vitis vinifera, XP_002265310.1), SICYP78A5-like (Solanum lycopersicum, XP_004236064.1), AtCYP78A5 (Arabidopsis thaliana, NP_172827.1), SICYP78A9 (Solanum lycopersicum, XP_004240114.1), SICYP78A3 (Solanum lycopersicum, XP_004230013.1), SICYP78A6-like (Solanum lycopersicum, XP_004248458.1), VvCYP78A3-like (Vitis vinifera, XP_002266493.1), TcCYP78A3 (Theobroma cacao, XP_017973443.1), VvCYP78A6 (Vitis vinifera, RVW14892.1), VvCYP78A9 (Vitis vinifera, RVW91651.1), AtCYP78A8 (Arabidopsis thaliana, NP_171627.2), AtCYP78A9 (NP_191747.1), AtCYP78A6 (Arabidopsis thaliana, NP_182189.1), PaCYP78A9-like (Prunus avium, XP_021815024.1), PbCYP78A6 (Pyrus bretschneideri, XP_009375445.2), MdCYP78A6-like (Malus domestica, XP_008343443.2), MdCYP78A9like (Malus domestica, XP_008343443.1). MEGA (version 5.10) was used to construct the phylogenetic tree with the Neighbor-joining method (1,000 replications bootstrap test and JTT model distribution). 
$\mathbf{A}$

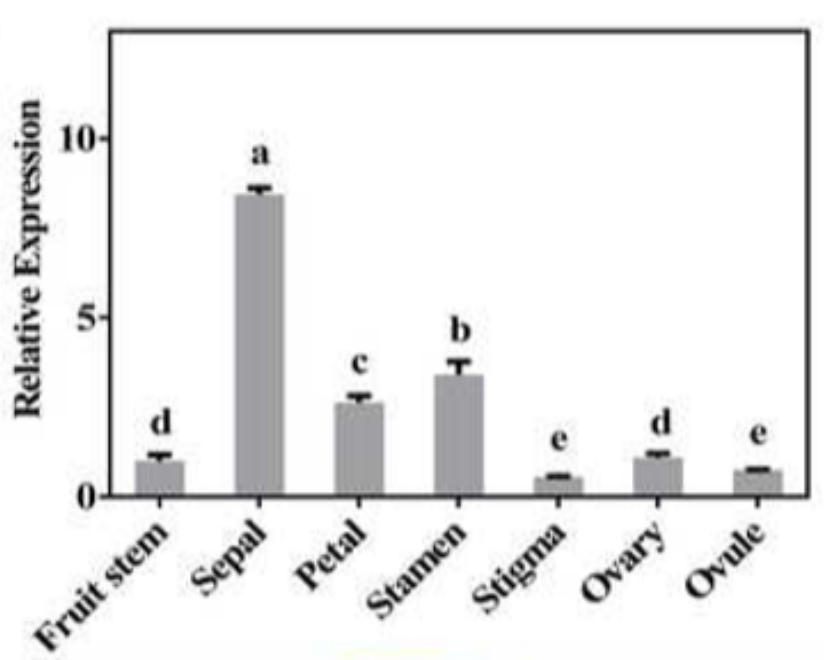

B

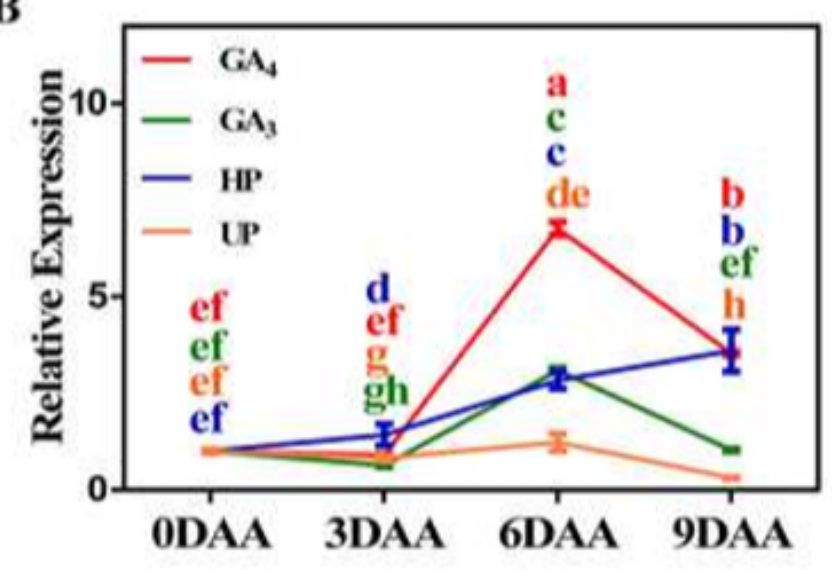

D

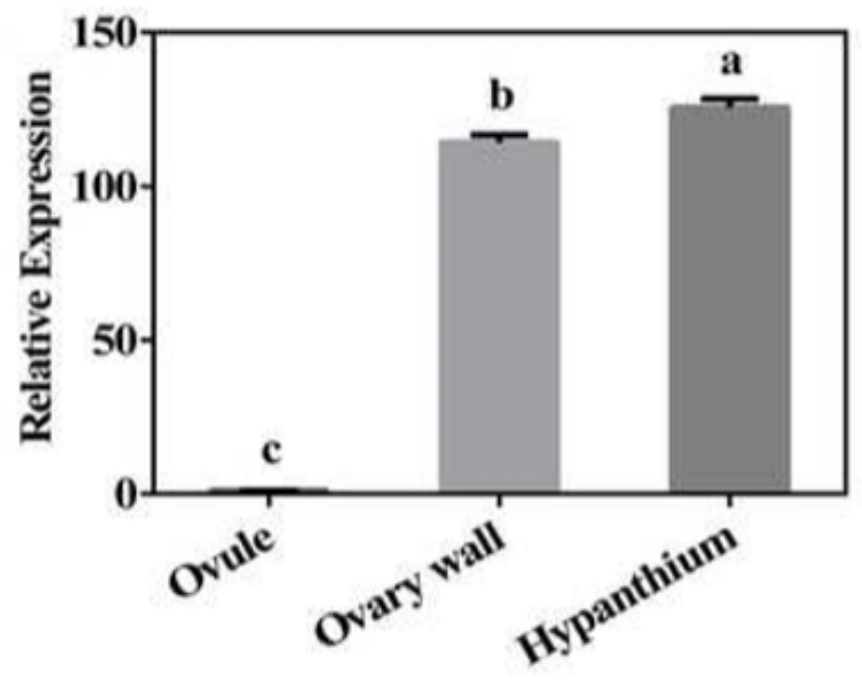

Figure 2

The PbCYP78A6 transcript levels in fruitlets at distinct developmental stages and in different tissues of 'Dangshansu' pear. A, The relative PbCYP78A6 expression levels were detected in reproductive tissues of 3-day-old pear flowers; B, The PbCYP78A6 expression patterns in 'Dangshansu' fruitlets at early distinct stages; $C$, Specific organs were sampled independently to detect the relative expression of PbCYP78A6 in a 30-day-old pear fruitlet; D, PbCYP78A6 transcript levels in different tissues of 'Dangshansu' fruitlets. An RT-qPCR analysis was used to determine the PbCYP78A6 transcript levels. UP: Un-pollinated; HP: Hand pollinated; GA4: treated with $50 \mathrm{mg} \mathrm{L-1} \mathrm{GA4;} \mathrm{GA3:} \mathrm{treated} \mathrm{with} 50 \mathrm{mg} \mathrm{L}-1$ GA3. DAA: days after anthesis. The results are represented as means of three biological replicates $( \pm S D s)$. Significant differences $(P<$ 0.05 ) among treatments as determined by a one-way ANOVA are indicated using different lowercase letters. 
A

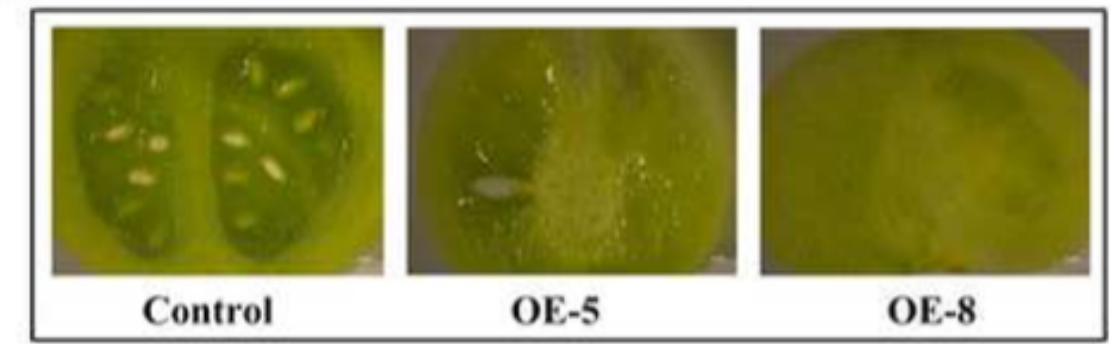

C

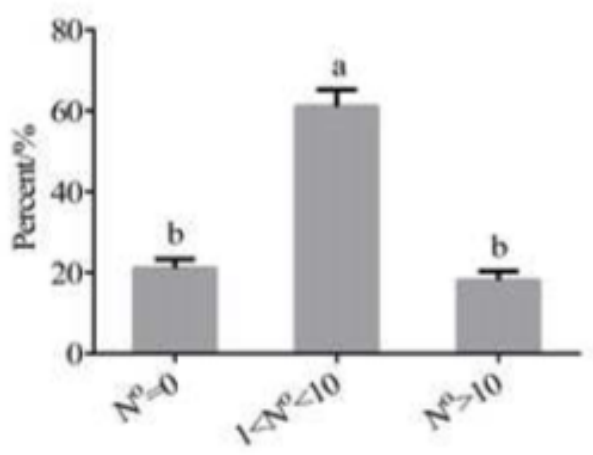

B

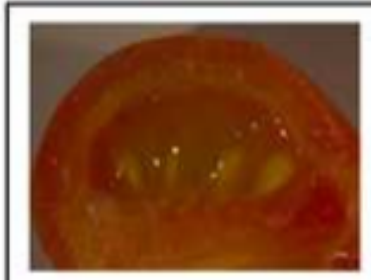

Control

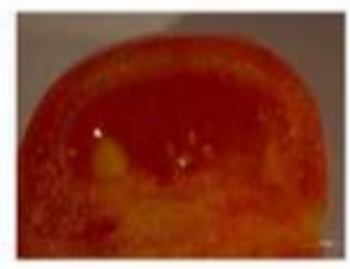

OE-5

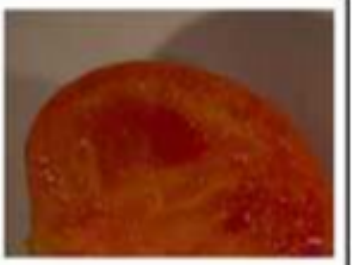

OE-8

D

E
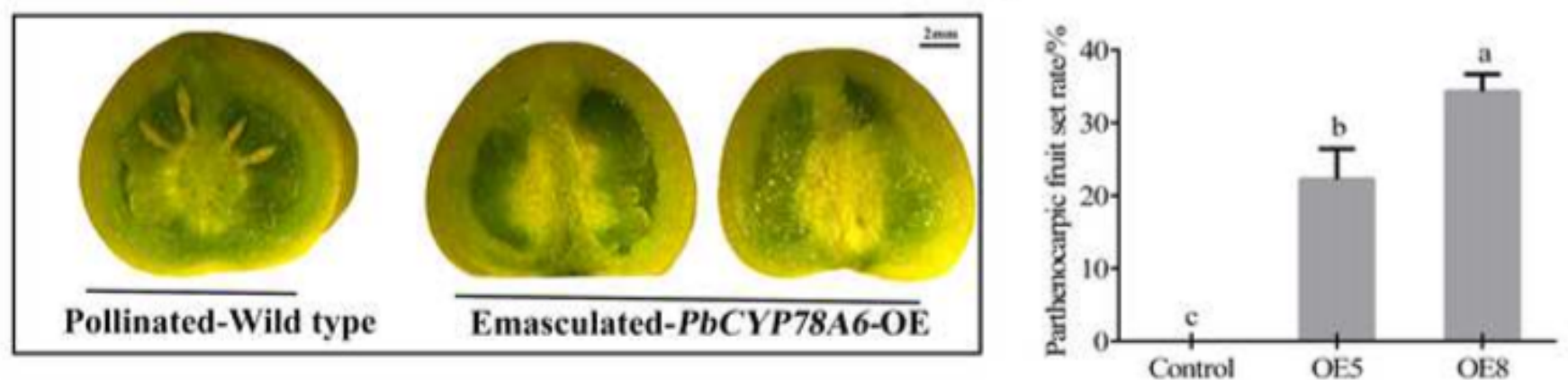

\section{Figure 3}

PbCYP78A6 overexpression contributed to reduced seed numbers and parthenocarpy in tomato. A, Longitudinal sections of wild-type and transgenic fruits produced by natural pollination at the green ripened stage; $B$, Longitudinal sections of wild-type and transgenic fruits produced by natural pollination at the ripened stage; $\mathrm{C}$, The percentages of PbCYP78A6-overexpression (OE) fruits containing different numbers of seeds under natural pollination conditions; $D$, Seeded fruit produced by hand pollination, and parthenocarpic fruit resulting from the emasculation of PbCYP78A6-OE lines; $E$, The parthenocarpic fruit set rates of transgenic PbCYP78A6-OE tomatoes. The results represented are means of three biological replicates $( \pm S D s)$. Significant differences $(P<0.05)$ among treatments as determined by a one-way ANOVA are indicated using different lowercase letters. Bars $=2 \mathrm{~mm}$ in $\mathrm{A}$ and $\mathrm{B}$. 

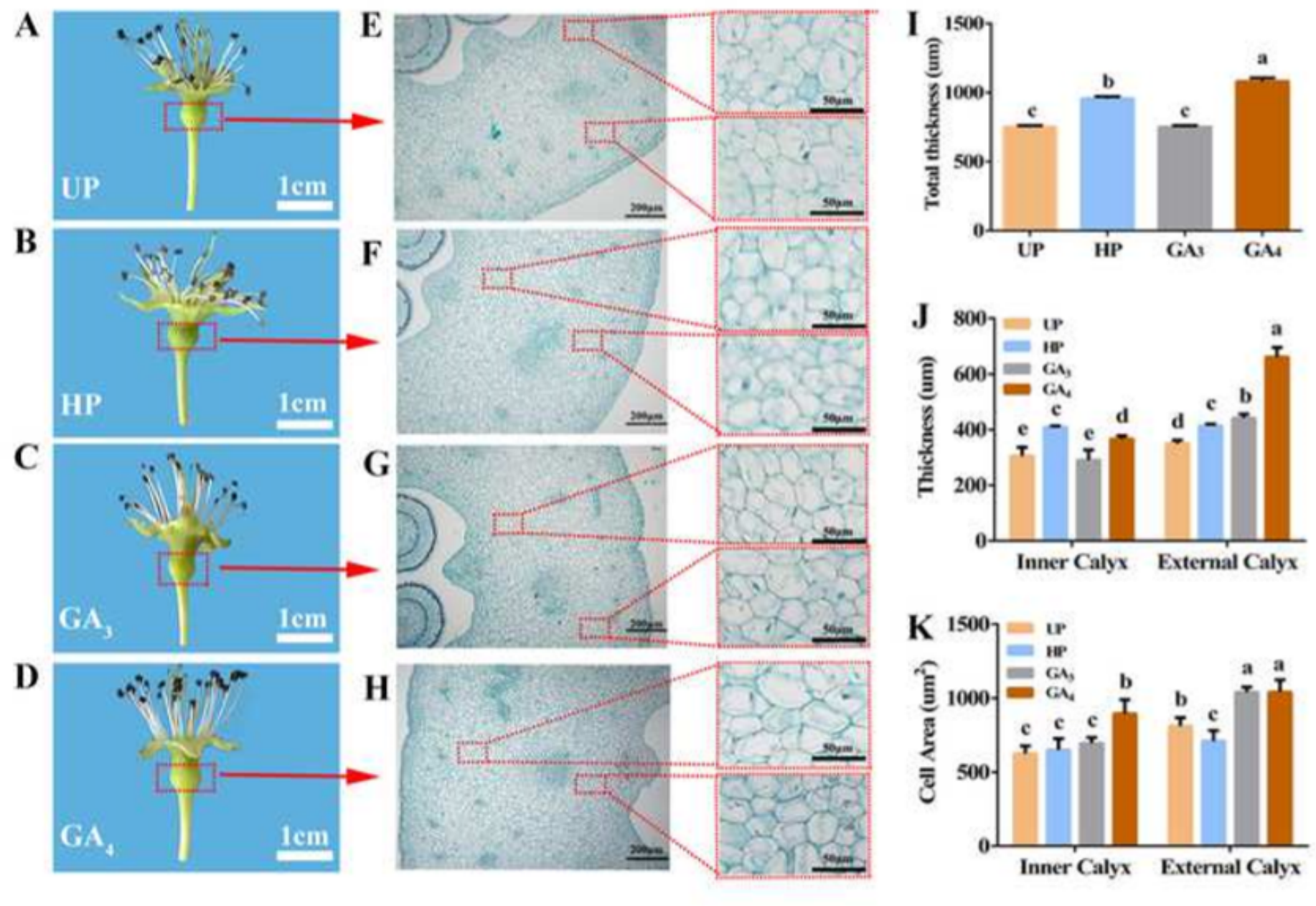

Figure 4

Phenotypic observations and histological features of 'Dangshansu' fruitlets 6 days after exposure to different treatments. A-D, phenotypes of UP, HP, and GA3- and GA4-treated 'Dangshansu' fruitlets at 6 DAA, respectively; $\mathrm{E}-\mathrm{H}$, histological observations of UP, HP, and GA3- and GA4-treated 'Dangshansu' fruitlets at 6 DAA, respectively; I, quantification of the total thicknesses of the fleshy calyxes in treated 'Dangshansu' pear; J, quantification of the internal and external thicknesses of the fleshy calyxes in treated 'Dangshansu' pear; $\mathrm{K}$, the cell areas of the internal and external fleshy calyxes in 'Dangshansu' pear. EC, External calyx (zone between the epidermis and the vascular bundle); IC, Internal calyx (zone inside the vascular bundle). The results represented are means of three biological replicates $( \pm S D s)$. Significant differences $(P<0.05)$ among treatments as determined by a one-way ANOVA are indicated using different lowercase letters. 


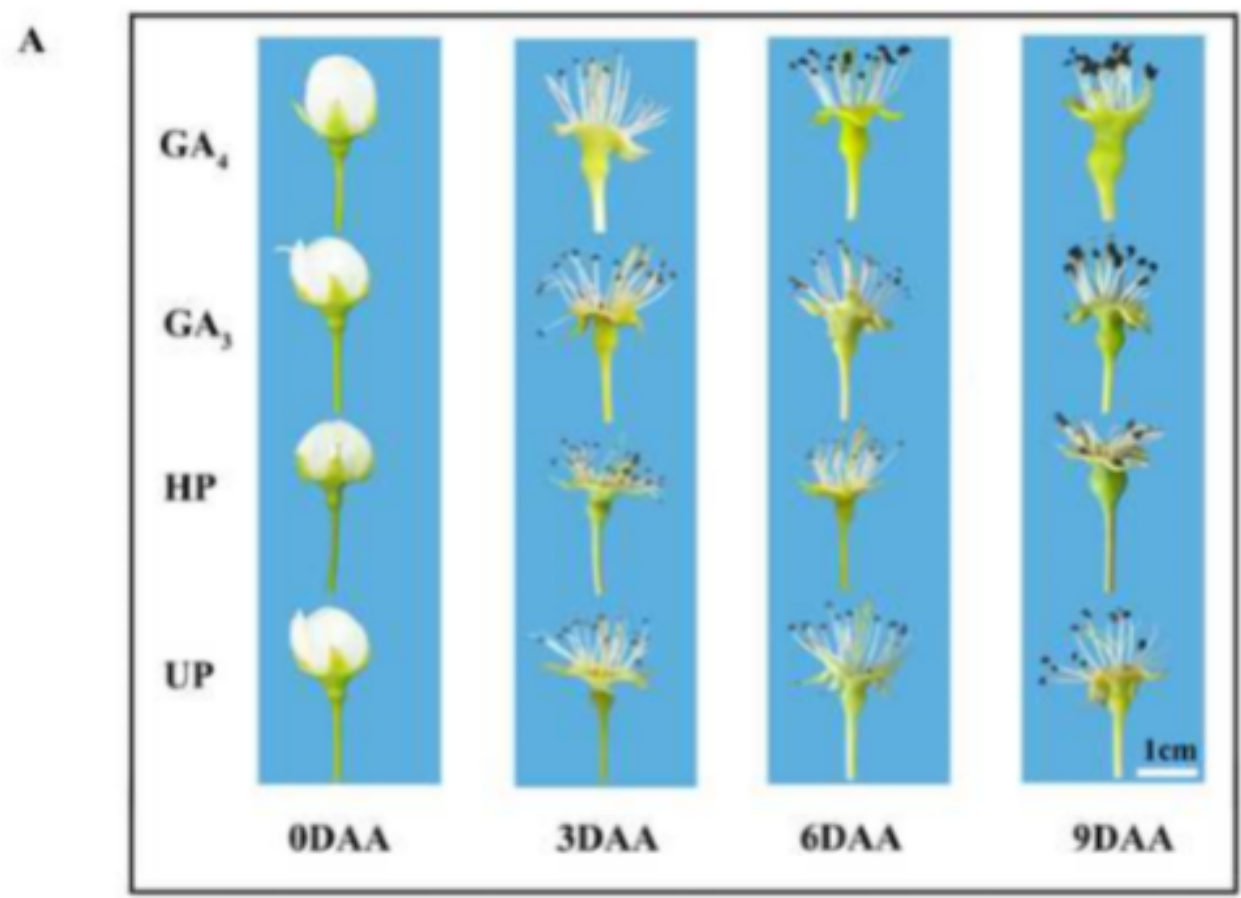

B
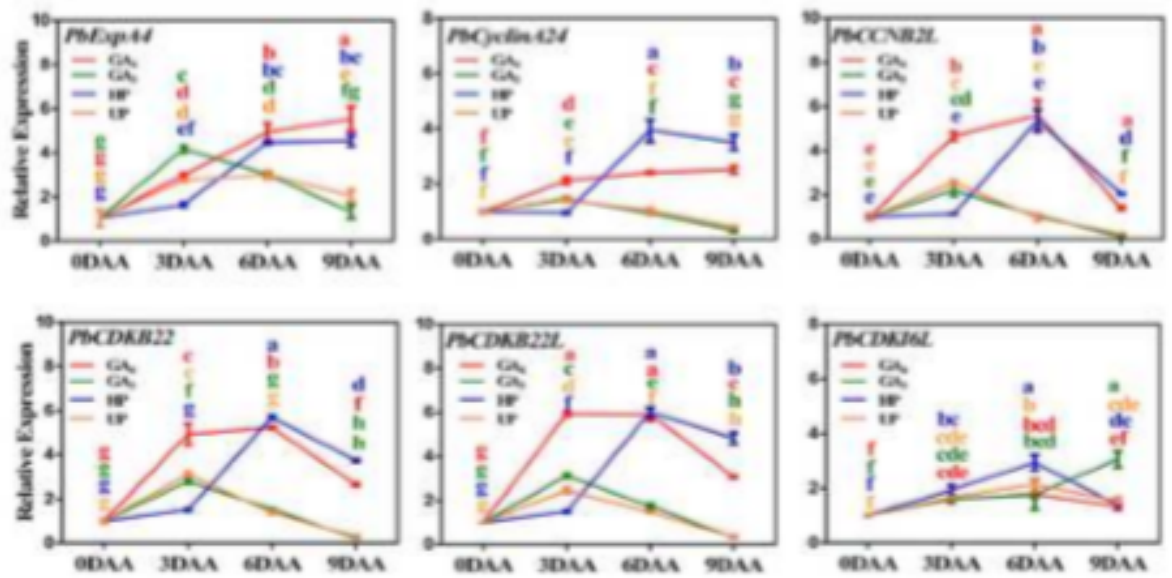

\section{Figure 5}

Dynamic growth of 'Dangshansu' fruitlets, and the transcript levels of cell cycle-related genes in 'Dangshansu' fruitlets at the early stage. A, The dynamic growth of 'Dangshansu' fruitlets after UP, HP, GA4 and GA3 treatments at 0, 3, 6 and 9 DAA; B, The expression levels of expansin-A4 (EXPA4), cyclinA24; G2/mitotic-specific cyclin-2-like (CCNB2L), cyclin-dependent kinase B2-2 (CDKB22), Pbcyclin-dependent kinase B2-2-like (CDKB22L) and cyclin-dependent kinase inhibitor 6-like (CDKI6L) in 'Dangshansu' fruitlets. The results represented are means of three biological replicates $( \pm S D s)$. Significant differences $(P<0.05)$ among treatments as determined by a one-way ANOVA are indicated using different lowercase letters. 
A
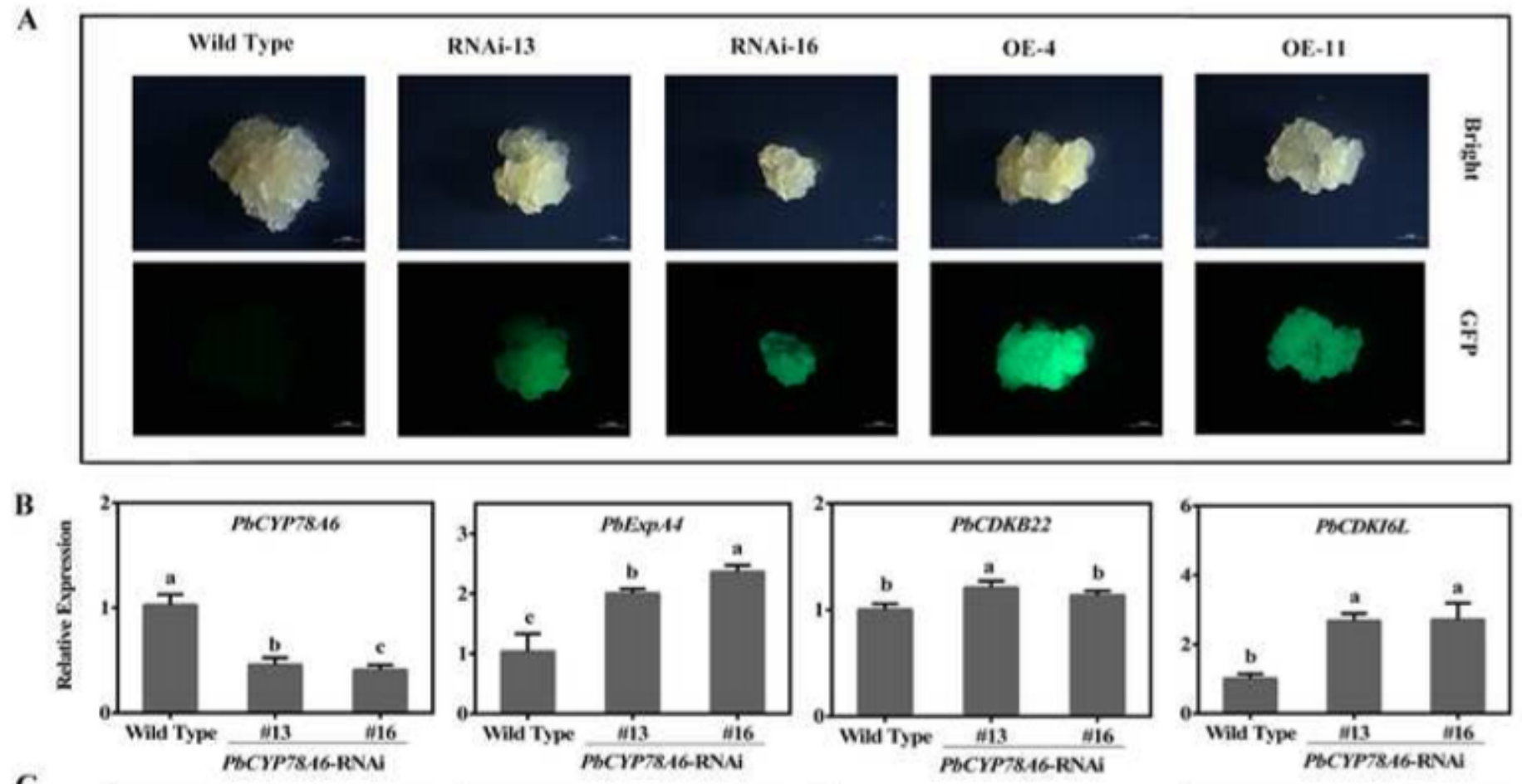

C
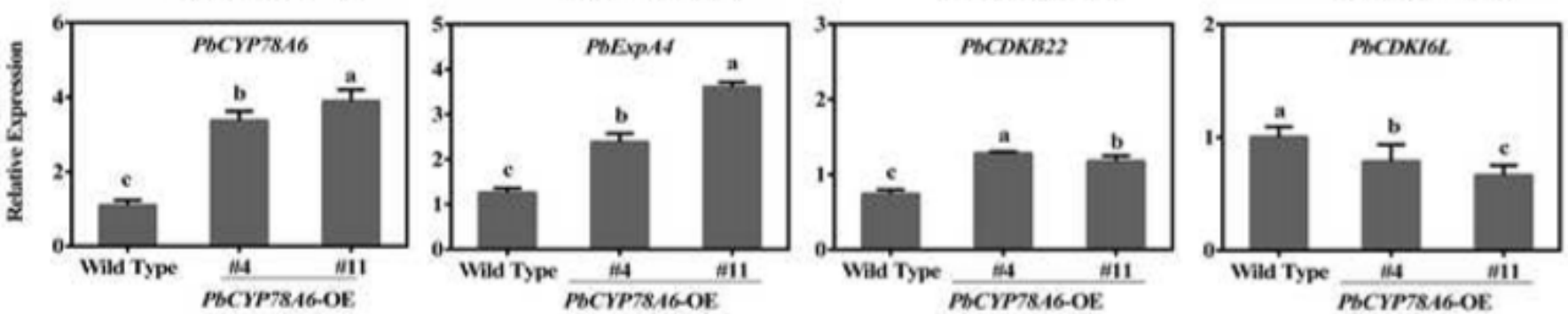

Figure 6

PbCYP78A6 promotes the expression of cell cycle-related genes in pear calli. A, Transgenic pear calli expressing green fluorescent protein (GFP) were detected. PbCYP78A6-RNAi, PbCYP78A6 RNA interference; PbCYP78A6-OE, PbCYP78A6 over-expression. B, RT-qPCR analysis of PbCYP78A6, PbEXPA4, PbCDKB22 and PbCDKI6L expression levels in PbCYP78A6-RNAi pear calli; C, Quantitative RT-PCR analysis of the PbCYP78A6, PbEXPA4, PbCDKB22 and PbCDKI6L expression levels in PbCYP78A6-OE pear calli. The results represented are means of three biological replicates $( \pm S D s)$. Significant differences $(P<0.05)$ among treatments as determined by a one-way ANOVA are indicated using different lowercase letters. 


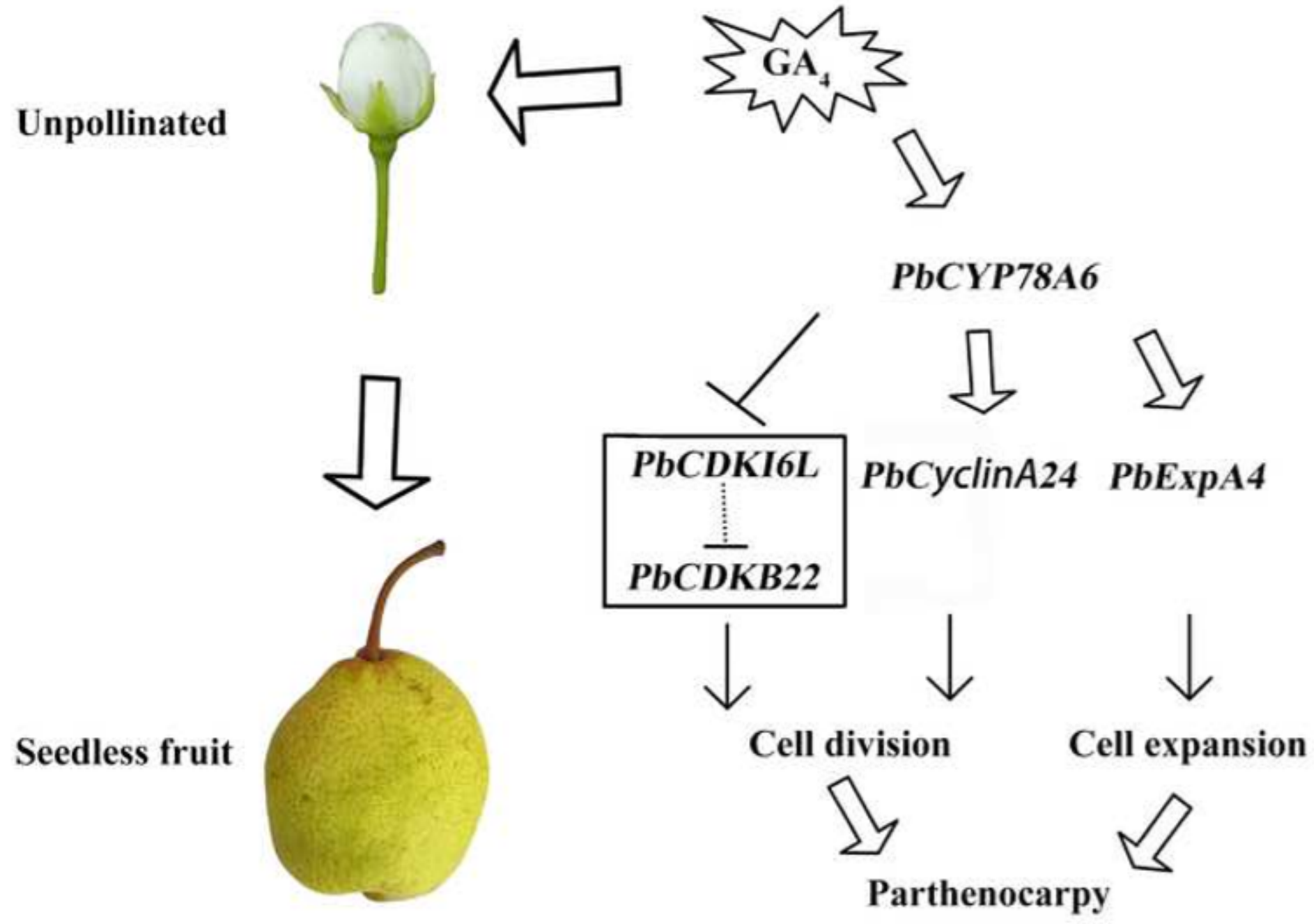

Figure 7

Model depicting the role of PbCYP78A6 in controlling fruit development-related genes to regulate parthenocarpy.

\section{Supplementary Files}

This is a list of supplementary files associated with this preprint. Click to download.

- Figs1.tif

- Figs21.tif

- Figs32.tif

- Figs41.tif

- FigS51.tif

- SupplementaryTableS1.xlsx 
- SupplepentaryTableS2.xIsx

- SupplementaryTableS3.xlsx

- SupplementaryTableS4.xIsx 